\title{
Angiostrongylus cantonensis: morphological and behavioral investigation within the freshwater snail Pomacea canaliculata
}

\author{
Shan Lv • Yi Zhang • He-Xiang Liu • Chao-Wei Zhang • \\ Peter Steinmann • Xiao-Nong Zhou • Jürg Utzinger
}

Received: 4 December 2008 / Accepted: 7 January 2009/Published online: 27 January 2009

(C) Springer-Verlag 2009

\begin{abstract}
An infection with Angiostrongylus cantonensis, the main causative agent for human eosinophilic encephalitis, can be acquired through the consumption of the freshwater snail Pomacea canaliculata. This snail also provides a suitable model to study the developmental morphology and behavior of $A$. cantonensis larvae, facilitated by the snail's distinct lung structure. We used microanatomy for studying the natural appearance and behavior of $A$. cantonensis larvae while developing within $P$. canaliculata. The distribution of refractile granules in the larval body and characteristic head structures changed during the developmental cycle. Two well-developed, rodlike structures with expanded knob-like tips at the anterior
\end{abstract}

\author{
S. Lv $\cdot$ P. Steinmann $\cdot$ J. Utzinger $(\bowtie)$ \\ Department of Public Health and Epidemiology, \\ Swiss Tropical Institute, \\ P.O. Box, CH-4002 Basel, Switzerland \\ e-mail: juerg.utzinger@unibas.ch \\ S. Lv \\ e-mail: 1vshan000@126.com \\ P. Steinmann \\ e-mail: peter.steinmann@unibas.ch \\ S. Lv $\cdot$ Y. Zhang $\cdot$ H.-X. Liu $\cdot$ C.-W. Zhang $\cdot$ P. Steinmann $\cdot$ \\ X.-N. Zhou \\ National Institute of Parasitic Diseases, \\ Chinese Center for Disease Control and Prevention, \\ 200025 Shanghai, People's Republic of China \\ Y. Zhang \\ e-mail: zhang1972003@yahoo.com.cn \\ H.-X. Liu \\ e-mail: hxliu17@hotmail.com \\ C.-W. Zhang \\ e-mail: zcw8025@yahoo.com.cn \\ X.-N. Zhou \\ e-mail: ipdzhouxn@sh163.net
}

part were observed under the buccal cavity as early as the late second developmental stage. A "T"-shaped structure at the anterior end and its tenacity distinguished the outer sheath from that shed during the second molting. Early first-stage larvae obtained from fresh rat feces are free moving and characterized by a coiled tail, whereas a mellifluous "Q"-movement was the behavioral trait of third-stage A. cantonensis larvae outside the host tissue. In combination, the distribution of refractive granules, distinct head features, variations in sheaths, and behavioral characteristics can be utilized for differentiation of larval stages, and for distinguishing $A$. cantonensis larvae from those of other free-living nematodes.

\section{Introduction}

The rat lungworm Angiostrongylus cantonensis is a zoonotic nematode endemic in different parts of the world (Kliks and Palumbo 1992). The majority of all human eosinophilic encephalitis cases in East Asia, Southeast Asia, the Pacific islands, and the Caribbean can be attributed to infections with this parasite (Wang et al. 2008). An infection with $A$. cantonensis usually results from the consumption of raw or undercooked mollusks and paratenic hosts harboring infectious third-stage larvae $\left(\mathrm{L}_{3}\right.$; Rosen et al. 1967; Tsai et al. 2001). An infection can also be acquired through the consumption of contaminated salad (Slom et al. 2002) and vegetable juice (Tsai et al. 2004). A wide variety of mollusks can serve as intermediate hosts for A. cantonensis ( $\mathrm{Lv}$ et al. 2008; Wang et al. 2008). Two species are of particular importance for transmission, namely, Achatina fulica and Pomacea canaliculata. A. fulica is a terrestrial snail, which plays an important role in the worldwide transmission of A. cantonensis (Kliks 
and Palumbo 1992; Caldeira et al. 2007). P. canaliculata is a big freshwater snail implicated in numerous case reports and outbreaks of eosinophilic encephalitis in East Asia and Southeast Asia, especially China (Tsai et al. 2001; Lv et al. 2008).

P. canaliculata was imported in Southeast Asia around 1980 with the aim of farming this snail as a source of protein. In the meantime, $P$. canaliculata has become widely distributed throughout the region (Joshi and Sebastian 2006). The snail has not only damaged crops and altered microenvironments, but has also facilitated the spread of A. cantonensis, since it has become a popular food dish in certain regions (Tsai et al. 2004; Lv et al. 2008).

There is a dearth of rapid and sensitive methods for the detection of $A$. cantonensis larvae within $P$. canaliculata. Recently, a novel detection method based on microanatomy has been presented (Liu et al. 2007). Conventional methods include artificial digestion, tissue fixation, and staining, and these approaches have been used to study the morphology of $A$. cantonensis and to identify its larvae. However, these conventional methods contain a number of shortcomings for the rapid detection of A. cantonensis in snails. For example, artificial digestion can destroy larval sheaths, which represent important criteria for stage identification. Worse, artificial digestion can even destroy the entire larval body. It is also important to note that most studies focused on the morphology of $\mathrm{L}_{3}$, while features unique to first-stage larvae $\left(\mathrm{L}_{1}\right)$ and second-stage larvae $\left(\mathrm{L}_{2}\right)$ were neglected. Fortunately, specific tissue structures of $P$. canaliculata allow recovering of the entire larvae by microanatomy, facilitating the systematic study of their morphology and behavior at different developmental stages. The objective of this study was to describe the morphology and locomotor behavior of $A$. cantonensis over the course of development within P. canaliculata.

\section{Materials and methods}

Parasite, snail, and experimental infection

$\mathrm{L}_{3}$ of $A$. cantonensis were obtained from naturally infected $P$. canaliculata in Lianjiang in Fujian province, southeast China. A total of five Sprague-Dawley (SD) rats were infected by intragastric injection of $50-80 \mathrm{~L}_{3}$ each. Thirtyfive days postinfection, fresh feces (i.e., not older than $12 \mathrm{~h}$ ) were collected from infected rats and $\mathrm{L}_{1}$ harvested by the Baermann technique (Barçante et al. 2003). Morphologically unambiguously identified A. cantonensis larvae (Hata and Kojima 1990) were then used to infect $P$. canaliculata for establishment of the parasite life cycle in the laboratory.

Eggs of $P$. canaliculata were obtained from Fuzhou in Fujian province, China and cultured in the laboratory to ascertain that developing snails remained uninfected throughout the study. A total of 80 adult snails (weight $>10 \mathrm{~g}$ ) were used for the experiment. The snails were fasted for $48 \mathrm{~h}$, and groups of five snails were exposed to a 200-ml suspension of $40,000 \mathrm{~L}_{1}$ in a dish (radius $=8.5 \mathrm{~cm}$, height $=6.5 \mathrm{~cm}$ ) for $12 \mathrm{~h}$ at room temperature. Subsequently, the snails were transferred to aquariums with clean water kept at a temperature of $21 \pm 1^{\circ} \mathrm{C}$ and fed a mixed diet consisting of vegetables and dried fish food.

\section{Morphological investigation}

Morphological investigation of developing A. cantonensis within $P$. canaliculata focused on the distribution of refractive granules, head features, and sheath structures. Refractive granules are defined as the refractive material in the larval body when observed under a microscope. To obtain undamaged larvae, we performed microanatomy under a binocular dissecting microscope. After experimental infection of the snails, every second day, $2 P$. canaliculata were sacrificed, and the lung was isolated to recover A. cantonensis larvae. The larval capsules on the lung walls are protuberant and therefore more obvious than in other tissues. Larvae randomly picked from these capsules were placed in a Petri dish filled with tap water, and morphological features of the larvae were studied under a microscope.

\section{Behavioral study}

In connection with the morphological investigations detailed above, the behavior of $A$. cantonensis larvae in the nodules of the snail lung was observed. Larval behavior can primarily be described with regard to the curvature of the larval body. The movement of larvae placed in Petri dishes was observed under a microscope fitted with a camera. Still pictures and video sequences were taken for documentation. For comparison, the behavior of free-living nematodes of the genus Rhabditis, isolated from water and soil, was also studied.

\section{Histological investigation}

In order to assess the usefulness of conventional histological cross-section for diagnosis of $\mathrm{L}_{3}$ larvae of A. cantonensis within snail tissue, conventional hematoxylin and eosin (HE) staining and histological cross-sections of snail tissue were performed on day 61 post-infection. For recovery of larvae, the lung and foot tissues of the snails were separated from the snail body, and equal amounts were allocated to traditional cross-sectioning and artificial digestion, respectively. A solution containing $0.2 \%$ pepsin and $0.7 \%$ hydrochloric acid was used for artificial digestion, at a temperature of $37^{\circ} \mathrm{C}$ and an incubation period of $2 \mathrm{~h}$ 
(Lv et al. 2006). Artificial digestion was used to identify the developmental stage of larvae within lung and foot tissues of the snail, and to ensure that all larvae indeed had developed into $\mathrm{L}_{3}$.

\section{Results}

\section{A. cantonensis morphology}

\section{Distribution of refractive granules}

A. cantonensis larvae recovered from lung tissue of P. canaliculata by means of microanatomy usually appeared to be intact. Few refractive granules were observed in very early stage $L_{1}$ (Fig. 1). At early-stage $L_{1}$ (Figs. 2 and 3), refractive granules began to emerge in the larval body, and in later-stage $\mathrm{L}_{1}$ (Figs. 4, 5, and 6) and $\mathrm{L}_{2}$ (Figs. 7 and 8), they had increased and even obscured the expanded gut. A clear line emerged at the esophagus-intestine junction, dividing the larval body into an anterior section with few refractive granules and a posterior section dense with granules. Later, just before the second molting, the line blurred, and the amount of refractive granules decreased (Fig. 9). Some big refractive granules could still be seen in the posterior section of early stage $\mathrm{L}_{3}$ (Figs. 10, 11, and 12). Late stage $\mathrm{L}_{3}$ were transparent, and the anus, excretory pore, and esophagusintestine junction could easily be identified (Fig. 13).
Fig. 1 Very early first-stage larva $\left(\mathrm{L}_{1}\right)$ of $A$. cantonensis recovered from fresh rat feces. The larva moves with a coiled tail (CT)Fig. 2 Early $\mathrm{L}_{1}$ of A. cantonensis recovered from P. canaliculata on day 3 postinfection. Unexpanded intestine $(U I)$ presents strand-like

Fig. 3 Early $\mathrm{L}_{1}$ of A. cantonensis recovered from $P$. canaliculata on day 5 postinfection. The intestine is subdivided into two segments: expanded intestine $(E I)$ at the anterior part and unexpanded intestine $(U I)$ at the posterior part

Fig. 4 Mid-stage $\mathrm{L}_{1}$ of A. cantonensis recovered from P. canaliculata on day 7 postinfection. The expanded intestine $(E I)$ replaces the unexpanded intestine observed at an earlier stage

Fig. 5 Late $\mathrm{L}_{1}$ of $A$. cantonensis recovered from $P$. canaliculata on day 11 postinfection. The intestine becomes obscure due to refractive granules, while the esophagus bulbus $(E B)$ anterior to the esophagus-intestine $(E I)$ line is clear, with wrinkles $(W)$ appearing at the anterior end Fig. 6 Late $\mathrm{L}_{1}$ of $A$. cantonensis recovered from $P$. canaliculata on day 15 postinfection. The body size has increased approximately to that of $\mathrm{L}_{2}$. The esophagus-intestine $(E I)$ line is clear due to heterogeneous distribution of refractive granules
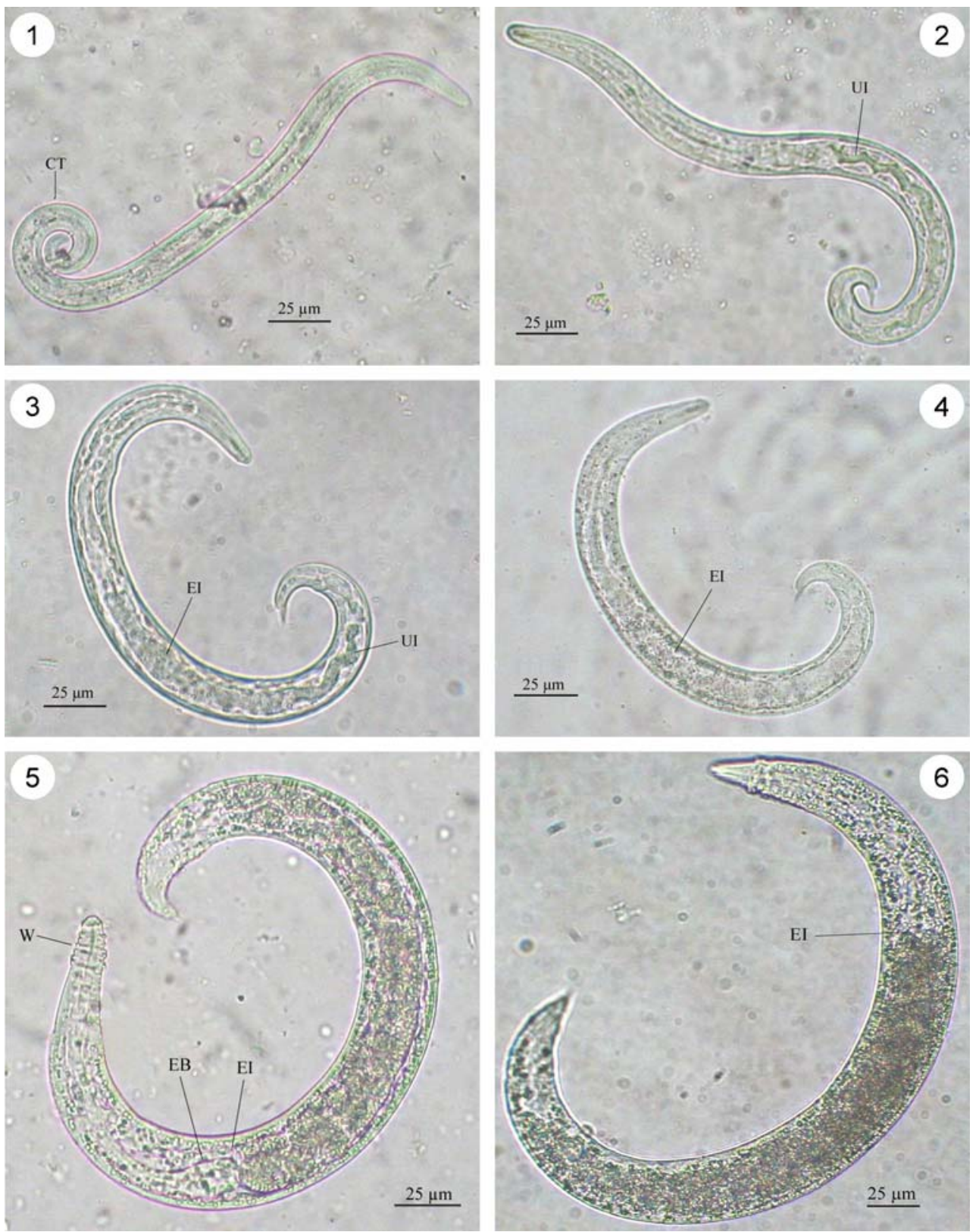
Fig. 7 Second-stage larva $\left(\mathrm{L}_{2}\right)$ of $A$. cantonensis with one sheath. The anus cuticle $(A C)$ of $\mathrm{L}_{1}$ is completely molted and presents on the sheath $(S)$. The esophagus-intestine $(E I)$ line appears clear

Fig. $8 \mathrm{~L}_{2}$ of $A$. cantonensis without sheath. The larval body is not destroyed, and the esophagus-intestine $(E I)$ line still appears clear

Fig. $9 \mathrm{~L}_{2}$ of $A$. cantonensis with expanded knob-like tips $(K T)$ and rod-like structure $(R S)$. The developed larva shows capability of movement and the esophagus-intestine line has disappeared, whereas the anus cuticle $(A C)$ of $\mathrm{L}_{1}$ is well presented on the sheath $(S)$

Fig. 10 Third-stage larva $\left(\mathrm{L}_{3}\right)$ of $A$. cantonensis with two sheaths. The outer one is the first sheath $(F S)$ produced during the first molting, whereas the inner one is the second sheath $(S S)$ produced during the second molting
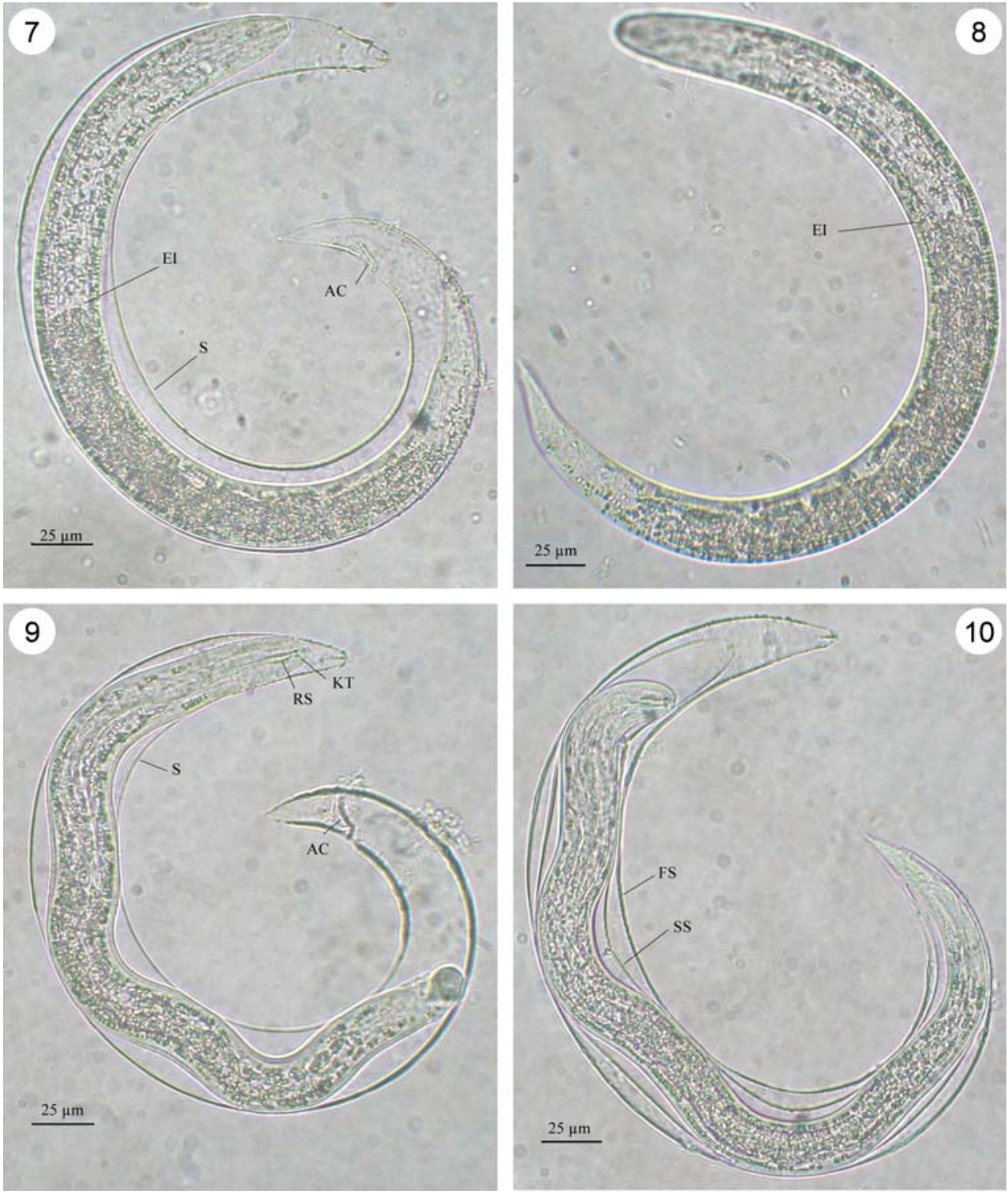

Ontogenesis of A. cantonensis larval head features

A pursed structure was observed at the apical end of the heads of early stage $\mathrm{L}_{1}$ of $A$. cantonensis (Fig. 14a). At a later stage of $\mathrm{L}_{1}$, the larval head had become wrinkled, and the head region appeared sharply conic. Additionally, a keratose cap covering the anterior end could be observed (Fig. 14b). $\mathrm{L}_{2}$ had a more obtuse and smooth head (Fig. 14c). Shortly before the second molting, the head features of $\mathrm{L}_{2}$ (Fig. 14d) were similar to that of $\mathrm{L}_{3}$ as shown in Fig. 14e: two welldeveloped, rod-like structures with expanded knob-like tips at their anterior end were observed under the buccal cavity.

\section{Variation in A. cantonensis larval sheaths}

Counting the number of sheaths is the standard method for differentiating developmental stages of nematodes
(Anderson 2000). The undisturbed sheaths of $A$. cantonensis larvae recovered from $P$. canaliculata by microanatomy confirmed that exsheathment did not occur while developing within the snails. However, the number of sheaths around larvae recovered from snail tissue by artificial digestion varied considerably. Some $\mathrm{L}_{3}$ had a single sheath sticking to their body surface through a vinculum-like structure (Fig. 11), while other larvae appeared to have no sheath at all (Figs. 12 and 13). Similar observations were made for $\mathrm{L}_{2}$ (Fig. 8).

Histological sections failed to clearly identify 2 sheaths for $L_{3}$. Typically, only one folded sheath could be observed (Figs. 15 and 16). There was no evidence suggesting that another sheath remained sticking to the wall of the cavity where the larvae had dwelled.

Microanatomy allowed observing the characteristics of sheaths shed during different moltings, resulting in several 
Fig. $11 \mathrm{~L}_{3}$ of $A$. cantonensis with a single sheath. The flexibility of this sheath forms a vinculum-like structure (VS) during larval movement

Fig. 12 Typical $\mathrm{L}_{3}$ of A. cantonensis with expanded knob-like tips $(K T)$ and rod-like structure $(R S)$, but no sheath

Fig. 13 Mature and transparent $\mathrm{L}_{3}$ of $A$. cantonensis. Clearly visible structures include excretory pore $(E P)$, esophagus bulbus $(E B)$, intestine $(I)$, and anus $(A)$

Fig. 14 Features of

A. cantonensis larval head at different stages of development; a head of early $\mathrm{L}_{1}$ with a pursed mouth $(P M)$; $\mathbf{b}$ head of late $\mathrm{L}_{1}$ with keratose cap $(K C)$ and wrinkles $(W)$; $\mathbf{c}$ head of $\mathrm{L}_{2}$ with a closed mouth $(M)$ and a "T"-shaped structure (TS) at the anterior end of the sheath; $\mathbf{d}$ larval head at the end of the second-stage with expanded knob-like tips $(K T)$ and rod-like structure $(R S)$, whereas a "T"shaped structure $(T S)$ is apparent at the anterior end of the sheath; e head of $\mathrm{L}_{3}$ with expanded knob-like tips $(K T)$ and rod-like structure $(R S)$
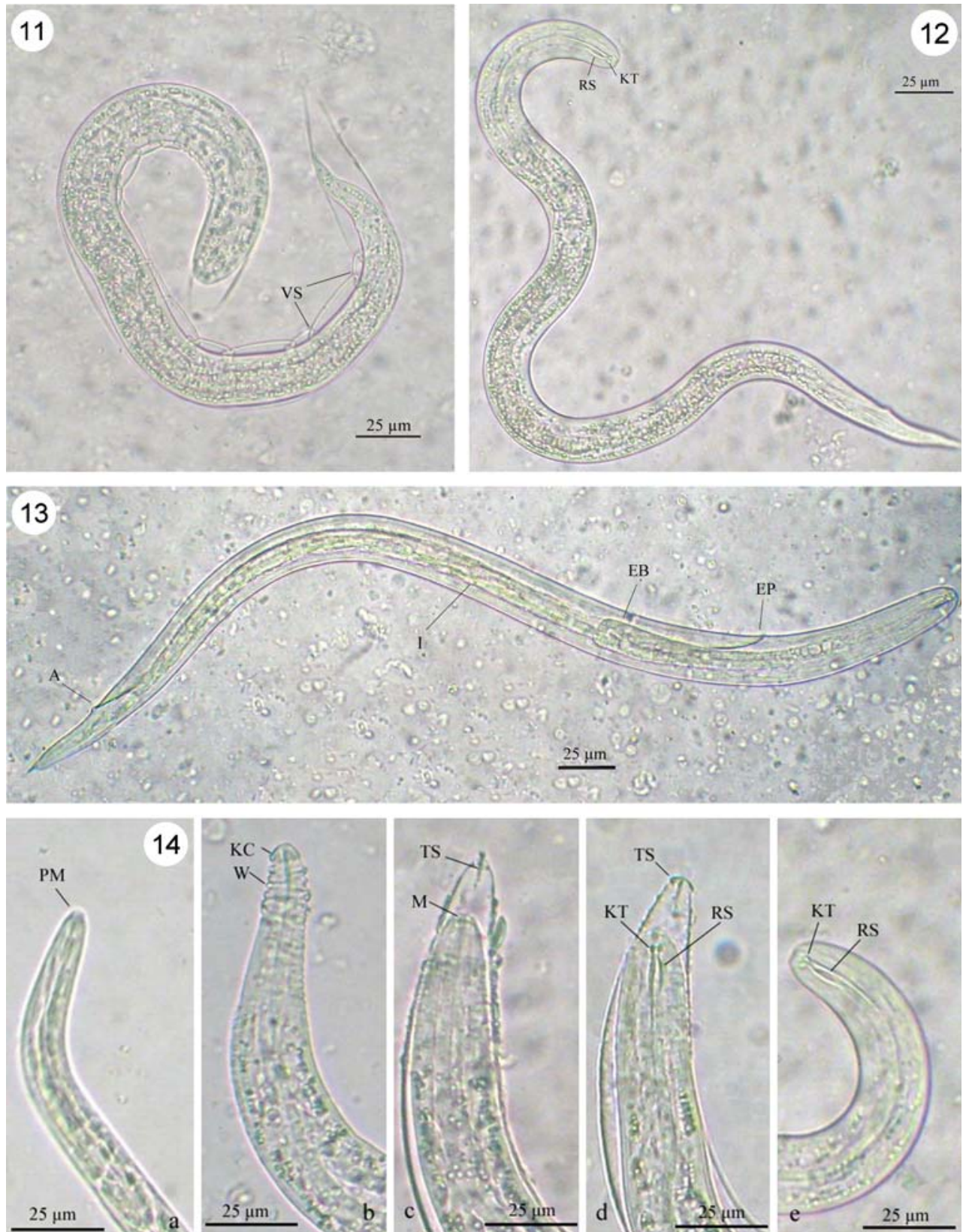

important observations. First, the inner sheath appeared more flexible than the outer one: while the former wrinkled when $\mathrm{L}_{3}$ moved, the latter seldom did so when larvae were observed shortly after recovery from the snail. This finding is supported by the observation that the outer sheath was rarely wrinkled when $\mathrm{L}_{2}$ wriggled. Second, a "T"-shaped structure (Figs. 10, 14c, and 14d) on the outer sheath distinguished the sheath shed during the first molting from that shed during the second one (Figs. 10 and 11). Third, the inner sheaths were more obtuse at the anterior part than the outer ones.

\section{Behavioral results}

\section{A. cantonensis movement outside the host}

Early stage $\mathrm{L}_{1}$ recovered from freshly collected rat feces usually kept their tail coiled, even when moving (Fig. 1), while the tail of larvae recovered from snails on the third day after infection was stretched. Early stage $\mathrm{L}_{1}$ were often fast moving, while middle-stage $\mathrm{L}_{1}$ usually appeared motionless and "C"-shaped (Figs. 3 and 4). In late-stage $\mathrm{L}_{1}$, only the head could be observed wiggling occasionally 
Fig. 15 Histological crosssections of an A. cantonensis larva embedded in the lung of $P$. canaliculata. Capsule $(C)$ consisting of host cells surrounds the larval body $(L B)$ with sheath $(S)$. The normal lung tissue $(L T)$ contains small cavities

Fig. 16 Histological crosssections of an A. cantonensis larva in the foot of $P$. canaliculata. The capsule $(C)$ containing the larval body $(L B)$ with sheath $(S)$ is embedded in the foot muscle $(F M)$ of the snail

Fig. 17 The "Q"-movement of $\mathrm{L}_{3}$ of $A$. cantonensis with integral sheaths outside the intermediate host $P$. canaliculata Fig. 18 The "Q"-movement of $\mathrm{L}_{3}$ of $A$. cantonensis without sheaths outside the intermediate host $P$. canaliculata

Fig. $19 \mathrm{~L}_{2}$ of $A$. cantonensis in a nodule on the lung wall of $P$. canaliculata. The snail tissue $(S T)$ is isolated from the lung. The esophagus-intestine (EI) line can be identified by the distribution of refractive granules and the head $(H)$

Fig. $20 \mathrm{~L}_{3}$ of $A$. cantonensis in a nodule on the lung wall of $P$. canaliculata. A typical capsule/nodule $(C)$ containing the coiling larval body $(L B)$ is embedded in the snail tissue $(S T)$
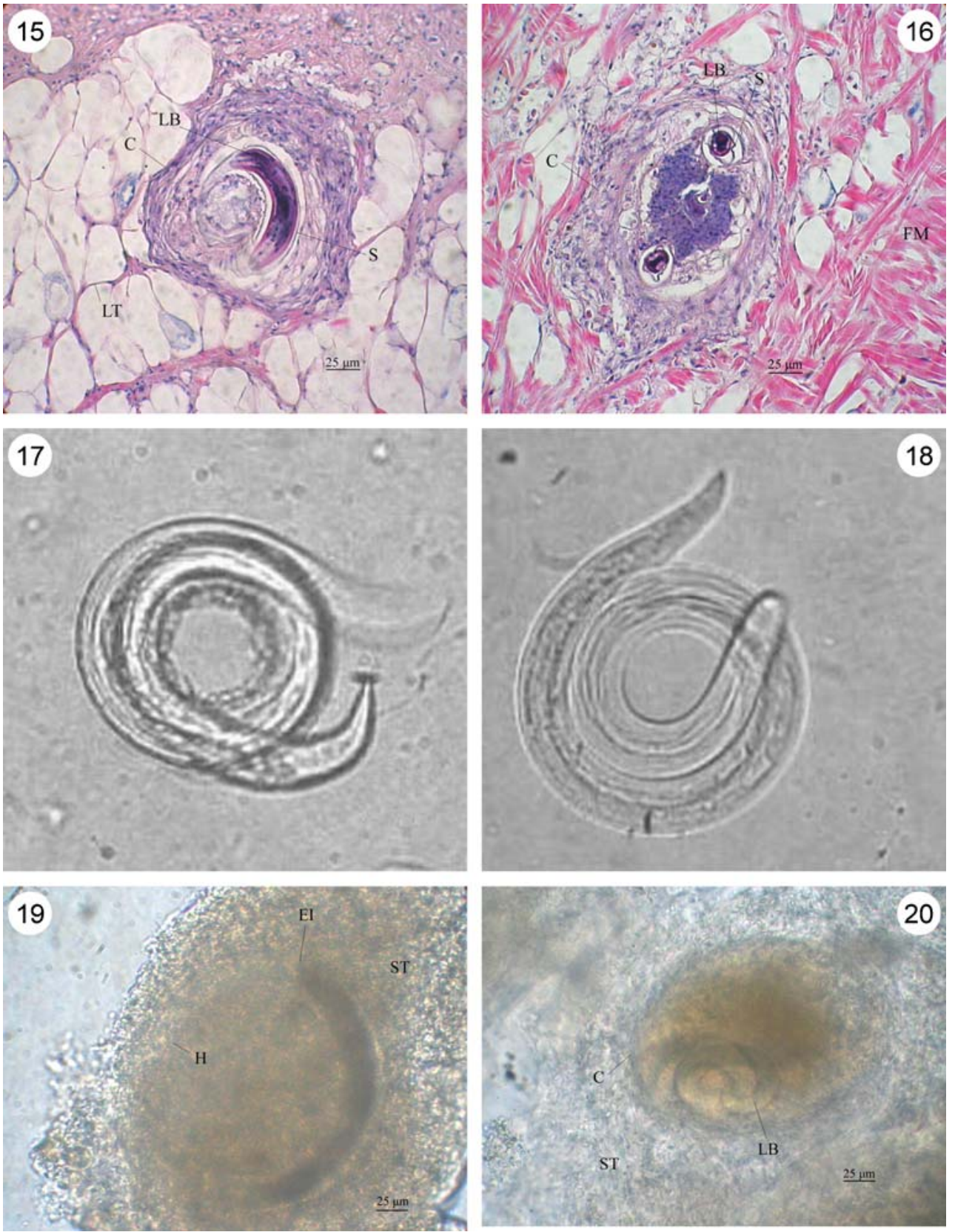

(Figs. 5 and 6). Due to the constraint presented by the sheath, $\mathrm{L}_{2}$ merely vermiculated inside the sheath (Figs. 7 and 9), but if unsheathed, they showed slow movement. A mellifluous "Q"-movement characteristic of $\mathrm{L}_{3}$ (Figs. 17 and 18) was embedded in regular "S"-movement. Although some early-stage $\mathrm{L}_{3}$ were still constrained by their sheaths, later stage $\mathrm{L}_{3}$ with two sheaths, as well as certain $\mathrm{L}_{3}$ with a single sheath, consistently showed the mentioned characteristic "Q"-movement. This behavioral trait was not observed in other free-living nematode species and, hence, seems distinctive for $A$. cantonensis.
Shape of A. cantonensis larvae within the host tissue

Since most larval nodules are deeply embedded in the host tissue, it is difficult to observe the shape of the larvae. However, the lung tissue of $P$. canaliculata allows the direct observation of $A$. cantonensis larvae harbored inside. $\mathrm{L}_{1}$ were observed to migrate to the lungs of $P$. canaliculata and to lodge in their walls, motionless, and in "C"-shape until undergoing further development. $\mathrm{L}_{2}$ (Fig. 19) started to coil and finally became tightly coiled $\mathrm{L}_{3}$ (Fig. 20). $\mathrm{L}_{3}$ embedded in lung tissue were enclosed in 
capsules and did not migrate, indicated by the absence of empty nodules.

\section{Discussion}

Eosinophilic meningitis, a potentially fatal human brain disease caused by the nematode $A$. cantonensis, is endemic throughout Southeast Asia and East Asia, the Pacific islands, and the Caribbean (Kliks and Palumbo 1992; Prociv et al. 2000; Waugh et al. 2005). This food-borne parasitic disease is emerging in China, most likely due to the expansion of P. canaliculata ( $\mathrm{Lv}$ et al. 2008, 2009). Detection techniques for the identification of contaminated food are an important tool for the control of eosinophilic meningitis. However, only few immunological and biomolecular diagnostic tools are currently available that have a high sensitivity and specificity (Qvarnstrom et al. 2007). Therefore, conventional techniques, most notably artificial digestion and microscopic examination, remain the methods of choice for diagnosis. Unfortunately, these approaches have several limitations. For example, freshwater and terrestrial snails and slugs are often contaminated with nonparasitic nematodes, rendering unambiguous species identification a prerequisite. Additionally, most studies focus on the morphology of infectious $\mathrm{L}_{3}$ of $A$. cantonensis, while $L_{1}$ and $L_{2}$ are often neglected (Ash 1970). Although the morphology of $A$. cantonensis in culture media has been described before (Hata and Kojima 1990), detailed investigation in the snail intermediate host has rarely been done. Artificial digestion is commonly used to recover larvae which reside deep inside the host tissue. During artificial digestion, the sheaths can be broken and their bodies damaged.

We developed an animal model for systematically studying the developmental morphology and behavior of A. cantonensis. Since $P$. canaliculata snails have a distinct lung structure (Giraud-Billoud et al. 2008), and the delicate wall of their lungs render larval nodules protuberant and visible, histological investigation can be performed. The nodules induced by $A$. cantonensis larvae in other parts of the snail body are more deeply embedded in the tissue and, hence, can rarely be identified under a microscope.

In the present study, particular attention was paid to the distribution of refractive granules, distinct head features of the larvae, variations in sheaths, and the larval locomotor behavior outside the snail host. Interestingly, the distribution of refractive granules has not been described in great detail before, despite this being one of the most obvious morphological characteristics to be studied under a microscope (Ash 1970; Bhaibulaya 1975; Brockelman et al. 1976; Hata and Kojima 1990). The distribution of refractive granules appeared characteristic for each larval stage of $A$. cantonensis and distinct from that observed in other free-living nematodes. Our observations suggest that the density and distribution of refractive granules could be employed for establishing criteria to distinguish $A$. cantonensis larvae from those of other nematodes, and for identifying different developmental stages. However, it is also necessary to deepen our understanding of the dynamics of refractive granules in the larval body. Recently, Hüttemann and colleagues (2007) employed transmission electron microscopy (TEM) and described the inner structure of adult $A$. cantonensis.

Head structures of $A$. cantonensis appeared to change during larval development. The structure at the anterior end of $\mathrm{L}_{1}$ obtained from rat feces consists of a closed mouth and sensory papillae, which can be observed by scanning electron microscopy (SEM; Xu et al. 1989; Ding et al. 1990; Hüttemann et al. 2007). The observed head features of late-stage $\mathrm{L}_{1}$ differed from previous descriptions (Alicata 1965; Hata and Kojima 1990). Wrinkles emerged at the anterior end of the head, implying that late-stage $\mathrm{L}_{1}$ can move their heads, suggesting that the larvae observed in the present study could have been further developed than those previously described. Rod-like structures with expanded knob-like tips at their anterior end are generally considered characteristic of $\mathrm{L}_{3}$ (Alicata 1965; Hata and Kojima 1990). Indeed, previous SEM investigations of $\mathrm{L}_{2}$ revealed that the mouth of this larval stage is similar to the closed mouth observed in $\mathrm{L}_{1}$ (Ding et al. 1990). However, in the present study, similar mouth structures were observed for $\mathrm{L}_{2}$ and $\mathrm{L}_{3}$. Thus, this specific feature should not be considered characteristic of $\mathrm{L}_{3}$ only.

The number of sheaths enclosing larvae is the main feature for nematode stage classification. However, intact larval sheaths can rarely be observed after artificial digestion since this procedure usually results in the loss of sheaths, and $\mathrm{L}_{2}$ without any sheath and $\mathrm{L}_{3}$ with a single sheath are commonly observed. In histological sections, only the folded inner sheath enclosing the $\mathrm{L}_{3}$ larval body can be observed. Microanatomy of larval nodules on lung walls allows studying intact sheaths. The sheaths generated during the first molting are tenacious with a " $\mathrm{T}$ "-shaped structure at the anterior end. The tenacious outer sheath might counteract the pressure from the snail tissue, since it forms a cavity within which the larvae lodge. This feature clearly distinguishes the sheath produced at first molting from that produced during the second molting. We speculate that the sheath features described here might facilitate an enhanced stage classification of $A$. cantonensis.

It is well known that nematode locomotor behavior varies from one species to another due to different structures and mechanisms (Gaugler and Bilgrami 2004). Yet, nematode locomotor behavior has been rarely utilized for species differentiation. The mellifluous "Q"-movement 
of $\mathrm{L}_{3}$ A. cantonensis larvae clearly distinguishes them from free-living nematodes. Hata and Kojima (1990) studied larvae cultured in vitro and noted that exsheathment could either be attributed to suppressing factors which were absent in the medium or to factors which facilitated exsheathment. Our observations indicate that $\mathrm{L}_{3}$ exsheath shortly after recovery from the host tissue when placed in tap water. We conjecture that physical factors might drive this process, and exsheathment is common outside the intermediate host.

The $P$. canaliculata model employed here allows to microscopically observe $A$. cantonensis larvae harbored in the nodules on the lung wall of the snail. The knowledge of typical larval shapes at different developmental stages facilitates the reading and interpretation of histological sections. Neither empty nodules nor nodules with exsheathed larvae were observed, indicating that migration only occurs in the early period before larvae settle and nodules develop. Thus, it appears that larvae do not exsheath in snails, contradicting the observations of Alicata (1965) and Brockelman et al. (1976). However, as of now, it cannot be ruled out that the situation in P. canaliculata differs from that in other intermediate host snails.

The morphological and behavioral characteristics of A. cantonensis investigated in this study can be microscopically observed without preliminary fixing and staining. Thus, these tools could be employed for screening snails for infection with $A$. cantonensis, hence could become an important issue when attempting to enhance food safety. Additionally, the time of infection can be established based on the described features characterizing different developmental stages of the larvae, facilitating the identification of the source of contamination, and the planning and monitoring of control strategies.

Acknowledgments This study received financial support from the Chinese Ministry of Science and Technology (grant no. 2003BA712A0901). Specimens were collected in Fujian province with the assistance of Drs. Jin-Xiang Lin and Li-Sha Li and their team at the Fujian Center for Disease Control and Prevention in Fuzhou. SL is the recipient of a stipend from the "Stipendienkommission für Nachwuchskräfte aus Entwicklungsländern" from the Canton of Basel-Stadt. PS and JU gratefully acknowledge support by the Swiss National Science Foundation (project no. PBBSP3-123193 for PS and project no. PPOOB-102883 and PPOOB-119129 for JU). The execution of the experiments described in this article complied with all applicable laws of the People's Republic of China.

\section{References}

Alicata JE (1965) Biology and distribution of the rat lungworm, Angiostrongylus cantonensis, and its relationship to eosinophilic meningoencephalitis and other neurological disorders of man and animals. Adv Parasitol 3:223-248
Anderson RC (2000) Nematode parasites of vertebrates: their development and transmission. CABI, Wallingford, UK, $650 \mathrm{pp}$

Ash LR (1970) Diagnostic morphology of the third-stage larvae of Angiostrongylus cantonensis, Angiostrongylus vasorum, Aelurostrongylus abstrusus, and Anafilaroides rostratus (Nematoda: Metastrongyloidea). J Parasitol 56:249-253

Barçante J, Barçante T, Dias S, Vieira L, Lima W, Negrão-Corrêa D (2003) A method to obtain axenic Angiostrongylus vasorum firststage larvae from dog feces. Parasitol Res 89:89-93

Bhaibulaya M (1975) Comparative studies on the life history of Angiostrongylus mackerrasae Bhaibulaya, 1968 and Angiostrongylus cantonensis (Chen, 1935). Int J Parasitol 5:7-20

Brockelman CR, Chusatayanond W, Baidikul V (1976) Growth and localization of Angiostrongylus cantonensis in the molluscan host, Achatina fulica. Southeast Asian J Trop Med Public Health $1: 30-37$

Caldeira RL, Mendonca CL, Goveia CO, Lenzi HL, Graeff-Teixeira C, Lima WS, Mota EM, Pecora IL, Medeiros AM, Carvalho Odos S (2007) First record of molluses naturally infected with Angiostrongylus cantonensis (Chen, 1935) (Nematoda: Metastrongylidae) in Brazil. Mem Inst Oswaldo Cruz 102:887-889

Ding BL, Xu SE, Shen HX, Lu XJ, Zhang DP (1990) Scanning electron microscopic observations on larvae and young adults of Angiostrongylus cantonensis. Chin J Parasitol Parasit Dis 8:291-294

Gaugler R, Bilgrami AL (2004) Nematode behaviour. CABI, Wallingford, UK, $432 \mathrm{pp}$

Giraud-Billoud M, Koch E, Vega IA, Gamarra-Luques C, CastroVazquez A (2008) Urate cells and tissues in the South American apple snail Pomacea canaliculata. J Molluscan Stud 74:259-266

Hata H, Kojima S (1990) Angiostrongylus cantonensis: in vitro cultivation from the first-stage to infective third-stage larvae. Exp Parasitol 70:476-482

Hüttemann M, Schmahl G, Mehlhorn H (2007) Light and electron microscopic studies on two nematodes, Angiostrongylus cantonensis and Trichuris muris, differing in their mode of nutrition. Parasitol Res 101(Suppl. 2):S225-S232

Joshi RC, Sebastian LS (2006) Global advances in ecology and management of golden apple snails. Philippine Rice Research Institute, Nueva Ecija, 588 pp

Kliks MM, Palumbo NE (1992) Eosinophilic meningitis beyond the Pacific Basin: the global dispersal of a peridomestic zoonosis caused by Angiostrongylus cantonensis, the nematode lungworm of rats. Soc Sci Med 34:199-212

Liu HX, Zhang Y, Lv S, Zhu D, Ang XH, Hu L, Zhou XN (2007) A comparative study of three methods in detecting Angiostrongylus cantonensis larvae in lung tissue of Pomacea canaliculata. Chin J Parasitol Parasit Dis 25:53-56

Lv S, Zhou XN, Zhang Y, Liu HX, Zhu D, Yin WG, Steinmann P, Wang XH, Jia TW (2006) The effect of temperature on the development of Angiostrongylus cantonensis (Chen 1935) in Pomacea canaliculata (Lamarck 1822). Parasitol Res 99:583-587

Lv S, Zhang Y, Steinmann P, Zhou XN (2008) Emerging angiostrongyliasis in mainland China. Emerg Infect Dis 14:161-164

Lv S, Zhang Y, Liu HX, Hu L, Yang K, Steinmann P, Chen Z, Wang LY, Utzinger J, Zhou XN (2009) Invasive snails and an emerging infectious disease: results from the first national survey on Angiostrongylus cantonensis in China. PLoS Negl Trop Dis 3: e369

Prociv P, Spratt DM, Carlisle MS (2000) Neuro-angiostrongyliasis: unresolved issues. Int J Parasitol 30:1295-1303

Qvarnstrom Y, Sullivan JJ, Bishop HS, Hollingsworth R, da Silva AJ (2007) PCR-based detection of Angiostrongylus cantonensis in tissue and mucus secretions from molluscan hosts. Appl Environ Microbiol 73:1415-1419

Rosen L, Loison G, Laigret J, Wallace GD (1967) Studies on eosinophilic meningitis. 3. Epidemiologic and clinical observations 
on Pacific islands and the possible etiologic role of Angiostrongylus cantonensis. Am J Epidemiol 85:17-44

Slom TJ, Cortese MM, Gerber SI, Jones RC, Holtz TH, Lopez AS, Zambrano CH, Sufit RL, Sakolvaree Y, Chaicumpa W (2002) An outbreak of eosinophilic meningitis caused by Angiostrongylus cantonensis in travelers returning from the Caribbean. N Engl J Med 346:668-675

Tsai HC, Liu YC, Kunin CM, Lee SS, Chen YS, Lin HH, Tsai TH, Lin WR, Huang CK, Yen MY, Yen CM (2001) Eosinophilic meningitis caused by Angiostrongylus cantonensis: report of 17 cases. Am J Med 111:109-114
Tsai HC, Lee SS, Huang CK, Yen CM, Chen ER, Liu YC (2004) Outbreak of eosinophilic meningitis associated with drinking raw vegetable juice in southern Taiwan. Am J Trop Med Hyg 71:222-226

Wang QP, Lai DH, Zhu XQ, Chen XG, Lun ZR (2008) Human angiostrongyliasis. Lancet Infect Dis 8:621-630

Waugh CA, Shafir S, Wise M, Robinson RD, Eberhard ML, Lindo JF (2005) Human Angiostrongylus cantonensis, Jamaica. Emerg Infect Dis 11:1977-1978

Xu S, Ding BL, Lu XJ, Shen HX, Zhang DP, Xiao YK (1989) Scanning electron microscopic observations on adults of Angiostrongylus cantonensis. Chin J Parasitol Parasit Dis 7:32-34 